\title{
Determining the Antibiotic Resistance of Bacterial Pathogens in Sexually Transmitted Diseases
}

\author{
Vică Mihaela Laura, Matei Horea Vladi and \\ Siserman Costel Vasile \\ Additional information is available at the end of the chapter
}

http://dx.doi.org/10.5772/67871

\begin{abstract}
Sexually transmitted diseases (STD) are among the most common infections worldwide. These bacterial infections have spread predominantly in the developing/underdeveloped countries, the most common being syphilis, gonorrhea and those induced by Chlamydia trachomatis, Ureaplasma urealyticum or Mycoplasma spp. Due to extensive usage of antibiotics in the recent past, these bacteria developed resistance to those commonly used for treatment, such resistant strains becoming a public health problem in a number of countries. It is well documented that bacterial STD agents are difficult to detect using standard culture media because these methods require special conditions and adequate nutrients. Antimicrobial susceptibility testing is, therefore, difficult to obtain in such cases. In recent years, genetic tests have been frequently employed in STD diagnosis. The study of genes that induce resistance to antibiotics using DNA isolated from these bacteria may prove to be a viable alternative. Genetic methods enable the DNA extraction from different biological samples, and both the presence of the bacteria and their resistance to one or more antibiotics can be determined from a single DNA sample. By studying the genes that induce antibiotic resistance and the plasmids that transfer such genes, the mechanism that leads to antibiotic resistance can be elucidated.
\end{abstract}

Keywords: STD pathogens, antibiotic resistance, genes

\section{Introduction}

Sexually transmitted diseases (STD) are caused by a wide variety of bacteria, viruses or parasites transferred from one human being to another primarily by vaginal, anal or oral sexual 
contact. Different STD can coexist or be simultaneously transmitted, the presence of any such infection increasing the risk of contracting other STD types. Prolonged, untreated infections with these pathogens can induce anexitis, endometritis, pelvic peritonitis or spontaneous abortions (in women) and urethritis, prostatitis or epididymitis (in men), respectively.

Reasons for STDs' recurrence in some sexually active women include the anatomy of the female sex, multiple partners or nonusage of condoms [1]. Such infections present an increasingly serious threat to global public health, especially for mothers or babies, causing severe complications such as cancer of the cervix, spontaneous abortions, premature births, low birth weight or infertility [2]. A number of studies concluded that ulcerative and nonulcerative STDs significantly increase HIV transmission, and drug resistance is complicating the fight against HIV [3]. The presence in the genital tract of such microbial pathogens may be due to a poor hygiene or risky behaviors as mentioned above [4].

Microorganisms make use of a wide range of mechanisms to resist to antibiotics and survive their attack. The emergence of antibiotic resistance is a natural biological phenomenon that occurs as a reaction to the use of antibiotics. Antibiotic resistance results from the bacteria ability to resist to antibiotics as they develop and multiply in media with high antibiotic concentration.

The main causes of antibiotic resistance are their abusive usage and the spread of resistant bacterial strains or genes bearing information able to induce resistance. There are multiple mechanisms leading to antibiotic resistance, one involving resistance plasmids carrying genes responsible for resistance to antibiotics [5, 6]. The phenomenon of antibiotic resistance can also develop via mutations or by acquiring resistance genes from other resistant bacteria [7-9].

Resistance to macrolides observed in the STD strains is often associated with mutations in the ribosomal protein genes [10,11]. It is assumed that mutations in a specific region of the $23 S$ rRNA play a major role in inducing resistance to macrolides as a result of their interaction with the ribosomes $[12,13]$.

Treatment failure because of bacterial resistance to the major groups of antibiotics as a result of extensive usage became a serious threat to human health. Increased usage of nucleic acid amplification tests (NAATs) in the diagnosis of STD leads to the elimination of culture-based techniques, depleting the number of live strains available for resistance testing. A lack of routine test-of-cure makes it impossible to distinguish treatment failure from reinfection [14]. The cost of health care for patients with resistant infections is higher due to longer duration of illness, additional tests and use of more expensive drugs.

It is obvious that the microorganisms' antimicrobial resistance is a dynamic phenomenon which points out the need for updated prevalence and susceptibility data from vast geographic areas being available for relevant institutions [4].

\section{Most common bacterial STD agents and their resistance to antibiotics}

STD infections are induced by a diversity of bacterial pathoges, including Neisseria gonorrhoeae, Mycoplasma hominis, Mycoplasma genitalium and Ureaplasma urealyticum. However, Chlamydia 
trachomatis is the leading cause of sexually transmitted bacterial infections worldwide and, in developing countries, the leading cause of preventable blindness [15].

C. trachomatis strains are obligate intracellular bacteria presenting a unique biphasic developmental cycle. Following the endocytosis of the small infectious bodies into the host cell and their inclusion into specialized vesicles, C. trachomatis strains differentiate into metabolically active reticular bodies and replicate until the inclusion fills the host cell. At this stage, the reticular bodies differentiate into elementary bodies and are being released from the cell to continue the infection [14].

Infection further leads to diseases such as cervicitis, pelvic inflammatory disease, urethritis, proctitis, lymphogranuloma venereum, trachoma or reactive arthritis. Transmission of STD pathoges is largely uncontrolled as over $70 \%$ of the women and $50 \%$ of the men infected with C. trachomatis are asymptomatic. "Silent" chronic infection has been recognized as a significant cause of infertility in women. Symptomatic individuals seeking medical care often do not benefit from tests for initial diagnosis or cure, largely due to inherent costs. Empirical antibiotics treatment is hastening the development of drug resistance, as has already occurred for a closely related species, Chlamydia suis, in which a tetracycline transposon was acquired from another pathogens residing in the guts of pigs [16].

C. trachomatis is a Gram-negative parasite causing a wide range of inflammations in the urogenital tract. Clinical isolates exhibiting resistance to azithromycin and associated with recurrent infections have been described previously [17]. While several reports of human chlamydial infections that do not respond to tetracycline or doxycycline were produced, no human pathogenic chlamydial strains that demonstrate stable tetracycline resistance have yet been isolated $[17,18]$.

Plasmids are small circular self-replicating DNA particles coexisting in the bacterial cells that are capable of exporting genetic information from the bacterial chromosome. They can transfer genes usually not present in the bacterial chromosome. In prokaryotic organisms, plasmids usually code for genes that facilitate survival of the bacteria in the environment. In chlamydia, apart from their participation in glycogen synthesis and encoding proteins responsible for their virulence, their role has yet to be clearly recognized. Resistance plasmids carry genes responsible for antibiotics resistance. Plasmids and bacteriophages can be employed in the diagnostics of chlamydioses. Plasmids are already used for detection of chlamydial infections. Bacteriophages could be used as therapeutic agents, potentially replacing antibiotics to address the increasing problem of antibiotic resistance among chlamydia. The easy transfer of infectious elementary bodies into other cells can prove to be a useful biotechnological tool in the treatment of chlamydioses, particularly in the case of concealed and latent infections, principally at the time of chlamydial resistance to antibiotics used by choice of treatment (tetracyclines) [5]. According to some authors, chlamydial plasmids are not conjugative plasmids, and they do not code antibiotic resistance and do not share the capacity for integration, except for the integrative plasmid revealed in C. suis, which is integrated in a genomic island together with some insertion sequences in the bacterial chromosome that encodes genes of resistance to tetracycline (tet C) [16].

Chlamydial infections have high recurrence rates among sexually active populations [19]. However, whether this recurrence rate for the disease is due to reinfection or to persistent infection with the same organism has been difficult to determine [20]. Immunity to chlamydial 
infections is type specific; once the initial infection was resolved, reinfection is believed to result from exposure to chlamydial strains differing in type from the initial infecting strain [21]. In contrast, persistent infections are considered those in which Chlamydia has entered a noninfectious state that is metabolically quiescent.

Chlamydial infections are very likely to recur even when appropriate drug therapy is in place. Most clinical failures occur due to reinfection or relapse as the bacterial phenotype deviates to persistent, non replicating antibiotic-resistant types able to revert to the typical reticulate body phenotype once treatment is complete [22, 23]. Administration of tetracyclines and macrolides, impeding bacterial translation by binding to the $30 \mathrm{~S}$ or $50 \mathrm{~S}$ ribosomal subunits, is viewed as the recommended first-line therapeutic regimen for chlamydial infections [24]. Clinical isolates from patients with recurrent $C$. trachomatis infection were documented to have significant resistance against macrolides $[25,7]$.

Other frequently diagnosed STD worldwide is gonorrhoea, exhibiting a recent rise in its global incidence. N. gonorrhoeae are Gram-negative facultative intracellular bacteria accounting for about three-quarters of urethral discharge cases among South Africa men presenting to primary healthcare clinics [8]. Chlamydia and gonorrhoea share several aspects of their disease outcomes, both being able to cause pelvic inflammatory disease and epithelial scarring which can lead to infertility. On the other hand, they can also be asymptomatic. However, $N$. gonorrhoeae infections tend to be more acute, with symptoms including purulent discharge and acute local inflammation. Rashes and septic arthritis may occur if the bacterium disseminates through the host [14].

While not required for individual patient management, laboratory investigations are instrumental in the choice of antimicrobial agents. Due to a high prevalence of fluoroquinoloneresistant $N$. gonorrhoeae strains observed in the last decade, first-line therapy was changed to single-dose oral cefixime. However, ciprofloxacin - the first line treatment option until about 2008 - is still recommended for the treatment of presumptive gonorrhoea in patients with a history of severe allergy to cephalosporins. The increasing prevalence of $N$. gonorrhoeae antimicrobial resistance has become a global public health problem as lesser abilities to develop N. gonorrhoeae cultures and to perform antimicrobial susceptibility testing make monitoring antimicrobial resistance rather difficult. A significant advantage to public health programs and selective patient management could be provided by the use of molecular assays for gonococcal antimicrobial resistance, particularly in the case of fluoroquinolones [8].

Resistance to all antimicrobial agents has developed in some N. gonorrhoeae strains. The global spread of multidrug-resistant $N$. gonorrhoeae is a growing concern, clinical treatment failures with the extended-spectrum cephalosporins (the last option for empirical first-line monotherapy) being recently reported. In many countries, dual antimicrobial therapy (ceftriaxone plus azithromycin) is the recommended first-line empirical treatment [9].

Mycoplasmas, the smallest free-living microorganisms, are another important source of STDs. They belong to the class of Mollicutes and were first described in the 1950s, following isolation from a male patient with nongonococcal urethritis [26]. In healthy sexually-active adults urogenital, mycoplasmas are part of the normal commensal flora of the genital tract. M. hominis 
and $U$. urealyticum are involved in a wide array of infectious diseases, in adults as well as in children. They are suspected of being the causative agents of nongonococcal urethritis, prenatal infections leading to pregnancy complications, infertility, bacterial vaginosis and pelvic inflammatory disease [27].

In contrast to other bacteria, Mycoplasma spp. and Ureaplasma spp. are not susceptible to penicillins, cephalosporins, vancomycin or rifampicin. Present data suggest that urogenital mycoplasmas are susceptible to agents interfering with protein synthesis, such as the tetracyclines, macrolides, aminoglycosides and chloramphenicol, but also to topoisomerase inhibitors such as the fluoroquinolones. Various mycoplasmal species may pose selective innate resistance to an antibiotic to which other species might be sensitive, for example, all M. hominis strains are resistant to erythromycin. Mycoplasma also can develop resistance to antibiotics to which they are usually considered sensitive [28].

As a major cause of nongonococcal urethritis in men, having been directly linked to cervicitis, endometritis, and pelvic inflammatory disease in women, M. genitalium holds a distinct position in the spectrum of emerging pathogenic bacteria for humans. It has been isolated from the respiratory tracts and synovial fluid of diseased individuals along with the human pathogen Mycoplasma pneumoniae [29-31]. M. genitalium is also the smallest known self-replicating cell, with a genome size of only $580 \mathrm{~kb}$, an aspect which imposes severe biosynthetic limitations [32].

Because of extreme difficulties in cultivating M. genitalium, many researchers have relied on the results of serological and polymerase chain reaction (PCR) assays to establish links between $M$. genitalium and human disease. Antimicrobial susceptibility testing is consequently rather difficult.

Various investigators noted that resistance to tetracyclines of the mycoplasmas' is on an uphill trend worldwide [33], a Tunisian study reporting resistance rates of 22.7 and $25 \%$ among ureaplasmas and M. hominis, respectively [34]. Even higher values (45\%) were reflected in an American study, in contrast to some European ones suggesting much lower figures [35]. Macrolides and lincosamides are the antibiotics widely used for $U$. urealyticum infections, especially among children and patients allergic to tetracyclines or quinolones. However, widespread macrolide resistance in $U$. urealyticum has been recently reported. In contrast, pristinamycin, a newly promoted macrolide, proved to be ineffective against $U$. urealyticum and M. hominis [36]. Still, for sensible patients, for example, premature neonates, one must carefully consider the potential toxicity of the antibiotics in attempting to remove such bacteria [37].

\section{Determining the antibiotics resistance of pathogen STD agents}

Although resistance to several antibiotics has been reported in vitro, no evidence that $C$. trachomatis-generally treated with a single dose of azithromycin-has developed such resistance were documented [38]. For both chlamydia and syphilis, no internationally agreed methods to assess minimum inhibitory concentrations (MIC) in vitro are standardized [39]. Consequently, assessment of antibiotic resistance relies but on the identification of treatment failures. 
The emergence of drug resistance in many pathogenic bacteria compromised severely the therapeutic utility of macrolides. The occurrence of macrolide resistance is yet undocumented for many bacterial pathogens. The various molecular mechanisms inducing bacterial resistance can be collectively characterized as involving drug efflux, drug inactivation or alterations in the drug target site. The probability of developing resistance depends on the types and quantities of drug to which these organisms are exposed [10]. For instance, shortly after erythromycin was introduced in therapy in the 1950s not only was resistance in bacterial pathogens observed, but erythromycin-resistant strains were found to be cross-resistant to all other macrolides and also to the chemically unrelated lincosamide and streptogramin B drugs [40].

Resistance to macrolides of the $C$. trachomatis strains is often associated with mutations in the ribosomal protein genes, particularly in L4 and L22, but also as well as with mutations in the peptidyl transferase region of domain $\mathrm{V}$ of the $23 \mathrm{~S}$ rRNA gene. The later region was demonstrated to play an important role in the interaction of the ribosome with macrolides [10, 11]. Studies on macrolide-resistant $C$. trachomatis clinical isolates led to the first finding of mutations in the peptidyl transferase region of the 23S rRNA gene [12].

Many strains of $C$. suis, a pathogen of pigs, express a stable tetracycline resistance phenotype. This resistance pattern was demonstrated to be associated with a resistance gene, tet $(C)$, in the chlamydial chromosome. In tetracycline-resistant C. suis strains, four-related genomic islands sharing significant nucleotide sequence identity with resistance plasmids carried by a variety of bacterial species were identified. These genomic islands provided the first examples of horizontally acquired DNA integrated into a natural isolate of chlamydiae [16].

Lateral gene transfer (LGT) is a means allowing C. trachomatis to generate variants of enhanced relative fitness, as suggested by the high frequency of between-strain genetic recombinants of C. trachomatis among isolates obtained from human STD. Although hampered by the development of the pathogen, experimental investigations on this phenomenon detected in vitro LGT between strains of $C$. trachomatis in vitro. Host cells were simultaneously infected with an ofloxacin-resistant $\left(O f x^{r}\right)$ mutant of a serovar L1 strain and a rifampin-resistant $\left(\right.$ Rif $\left.^{r}\right)$ mutant of a serovar D strain. DNA sequencing was used to map genetic crossovers. Development occurred in the absence of antibiotics, the progeny being subjected to selection for $O f x^{r}$ Rif $^{r}$ recombinants. Natural DNA transformation is a plausible mechanism, although trans-DNA lengths were previously associated only with conjugation in known microbial LGT systems [41]. Nowadays, LGT studies can be performed with various other C. trachomatis combinations to study the mechanisms by which these strains can transfer resistance genes.

As for C. trachomatis no reliable laboratory-based gene transfer system was available, in vitro generation of recombinants from antibiotic-resistant strains was used to study the LGT, essential for generating between-strain genomic recombinants of $C$. trachomatis able to facilitate the organism's evolution [42]. For 16 in vitro-derived recombinants of ofloxacin- and rifampin-resistant L1 and D strains, multiple loci were examined and compared with the same sequenced loci among 11 clinical recombinants. Phylogenetics and bioinformatics were used in examining breakpoints and recombination frequency. Without any misclassification, in vitro and clinical isolates clustered perfectly into two groups using Ward's minimum variance based on breakpoint data. gyr A (confering ofloxacin resistance) and rpoB (confering rifampin 
resistance), but $\operatorname{trp} A$ as well, presented significantly more breakpoints among in vitro recombinants than among clinical recombinants. Significant selections were evidenced at other loci as well, results indicating that the in vitro model is statistically different from any natural recombination events. Additional genomic studies to determine the responsible factors for selection biases at unexpected loci are needed to clarify whether these are important for LGT approaches in the genetical manipulation of C. trachomatis [42].

Several genetic mechanisms are employed by pathogenic microbes for producing variants to counter host defenses. Such a high proportion of urogenital tract isolates presenting amino acid substitutions in the polymorphic ompA gene which encodes the major outer membrane protein (MOMP) suggests a role for spontaneous mutation followed by in vivo selection in the bacteria's route to avoid human immune defenses $[43,44]$. Humans have diverse $B$ and T cellmediated responses to MOMP, the occurrence of mutations in the same ompA segments of multiple clinical isolates suggesting the protectiveness of at least some of these responses [45]. Evidences also indicate that in vitro LGT may contribute significantly to the origin of enhanced-fitness variants in C. trachomatis [41]. The LGT mechanism has not been identified, but various publications investigating possible relationships between in vitro LGT and the high frequency of LGT recombinants in clinical isolates [46] suggested that a certain percentage of the many millions of clinical infections involve more than one strain of $C$. trachomatis. However, a recent study failed to identify mutations in the 23S rRNA genes of resistant mutants selected following enrichment by serial passage in the presence of subinhibitory concentrations of azithromycin [47].

Investigating mutations in the $23 \mathrm{~S}$ rRNA gene of macrolide-resistant isolates of wild-type C. trachomatis obtained from clinical samples and mutant strains selected using subinhibitory concentrations of the macrolides were the objective of another recent study [13] in which a set of resistant clinical isolates of $C$. trachomatis obtained from patients attending the Tianjin Institute of Venerology (Tianjin, China) during 20052008 was differentiated into wild-type and mutant strains, the 23S rRNA mutations in the isolates then being identified. Each patient was sampled for only one isolate, the antimicrobial agents examined being erythromycin, azithromycin and josamycin. The in vitro MICs of antibiotics in the isolated clinical strains of $C$. trachomatis were determined. As the MIC values of erythromycin in the eight strains of C. trachomatis were higher than the in-blood erythromycin concentration $(1 \mu \mathrm{g} / \mathrm{ml})$ and even higher than the tissue concentration of erythromycin in the urogenital system, these were considered to be wild-type macrolide-resistant strains of C. trachomatis.

Following PCR amplification of the $23 \mathrm{~S}$ rRNA gene of the eight wild-type resistant isolates (exhibiting MIC values above the tissue concentration of the antibiotic present in the urogenital system), no resistance-associated mutations were found at 2057 (E. Coli numbering scheme), 2058 or 2059, while only three resistant isolates presented the T2611C mutation. The isolates included the T2611C mutation in the case of two patients with persistent infection, but no mutations were found at 2058. A2057G mutations were found in six mutant isolates, while T2611C mutations were found in 10 mutant isolates. Two mutant resistant isolates presented A2059G mutations, while two of the resistant isolates exhibited no mutations in their $23 \mathrm{~S}$ rRNA sequences. Medical records indicated no response to azithromycin on behalf 
of the patients infected with mutant strains. The sensitivity of the wild-type clinical isolates to erythromycin and azithromycin was found to be lower than previously reported [7], explaining the high recurrence rate and treatment failure reported for chlamydial infections. Azithromycin treatment was unsuccessful in eight resistant strains. The wild-type resistant strains presented no mutations in the 235 rRNA, suggesting that other molecular mechanisms were responsible for their resistance. Possible mechanisms underlying drug resistance should be investigated in the future in order to understand the resistance of isolates exhibiting no mutation in the peptidyl transferase region of the $23 \mathrm{~S}$ rRNA gene. The merits of the Chinese study are that, for the first time, wild-type macrolide-resistant $C$. trachomatis strains have been observed in vitro, and for the first time, A2057G and A2059G mutations in the peptidyl transferase region of the 23S rRNA gene have been found in C. trachomatis with selective macrolide resistance [13].

While certain other studies reported that mutations observed in clinical strains were also found in laboratory strains, the reverse was found not to be true likely because rRNA mutations leading to drug resistance in a clinical pathogen often become apparent only when a drug therapy fails to eradicate that pathogen [10].

The first reported cases of clinically significant C. trachomatis infection resistant to ofloxacin and azithromycin came from a case study of two patients with $C$. trachomatis infections demonstrating multidrug resistance as they persisted after standard treatment [17]. A commercially available PCR test (Amplicor PCR; Roche Diagnostics, Indianapolis) was employed to detect $C$. trachomatis in urine and urethral/cervical swab specimens. Antimicrobial susceptibility testing on C. trachomatis strains isolated from cultured cells originating from urethral/ cervical swab specimens was conducted for doxycycline, azithromycin and ofloxacin. In vitro antimicrobial susceptibility testing was also completed.

The MIC (antibiotic concentration level where fluorescent antibody staining failed to highlight typical inclusions after incubation in cell culture) and the minimum chlamydicidal concentration allowing no inclusions on passage in an antibiotic-free medium (MCC) of these antimicrobial agents for these isolates were determined after a subsequent passage of the contents of duplicate unstained wells to a fresh monolayer in antibiotic-free medium. C. trachomatis DNA samples prepared from endocervical or urethral swab specimens were amplified by a nested PCR assay, amplicons being purified with a PCR purification kit (Qiagen, Chatsworth, CA). Sequencing was conducted on a model 377 (Perkin Elmer Biosystems, Foster City, CA) automated sequencer using a dRhodamine Terminator Cycle Sequencing kit according to the manufacturer's instructions. Edited sequences were aligned and analyzed with the GCG (Genetic Computer Group, Madison, WI) software package. Genotypes were determined by comparison of resulting sequences with reference $C$. trachomatis omp1 sequences in the GenBank database. Conclusions were that the mechanism responsible for heterotypic resistance in C. trachomatis remains unknown, being possible that the observed multidrug resistance to be phenotypic rather than genotypic in nature since the molecular targets of azithromycin, doxycycline and ofloxacin are quite different, and it is highly unlikely that a single or a limited number of gene mutation(s) could be responsible for simultaneous resistance to these diverse agents [13, 48]. 
Fosfomycin is a broad-spectrum antibiotic that irreversibly binds to the active site of murA. Fosfomycin renders the enzyme inactive by forming a covalent adduct with a cysteine residue in the active murA site [49]. It was demonstrated [50] that C. trachomatis can be resistant to high concentrations of fosfomycin. The genome sequences of $C$. trachomatis contain gene homologues in the peptidoglycan (PG) biosynthesis pathway, including murA. The study aimed to demonstrate in vitro activity of Chlamydia mur $A$ and in vivo activity in E. coli and to determine whether murA mRNA was expressed in Chlamydia bacteria at any time during the chlamydial developmental cycle. The murA gene from $C$. trachomatis serovar L2 was cloned and placed under the control of the arabinose-inducible, glucose-repressible ara promoter and transformed into Escherichia coli. Findings were that the expression of $C$. trachomatis mur $A$ mRNA is cell cycle dependent. Collectively, the data support the notion that Chlamydia organisms contain PG and suggest that PG in Chlamydia plays a role in development and division. Elucidating the existence of PG in Chlamydia spp. is significant for the development of novel antibiotics targeting the chlamydial cell wall.

Another study [51] made use of antibiotic resistance assays and whole genome sequencing to interrogate the hypothesis that two clinical isolates (IU824 and IU888) have acquired certain antibiotic resistance mechanisms (tetracycline efflux mediated by the presence of one of the 28 genes, ribosomal protection via carriage of one of the 10 tet genes or one otr gene, enzymatic inactivation conferred by one of the three tet genes or mutations in the 16S rRNA geneidentified as lending tetracycline resistance). The genes conferring the first three resistance mechanisms reside in plasmids and insertion elements (horizontally transmissible elements enabling their transfer among a wide range of bacterial species). No tetracycline resistance was evidenced in the two investigated strains, comparisons of the genome and plasmid sequences failing to identify regions able to accommodate horizontally acquired resistance genes.

Inability to introduce stably maintained DNA and perform targeted gene manipulation are long-standing limitations slowing progresses in understanding many of the components associated with the basic biology and pathogenesis of Chlamydia. One of these limitations was removed when a novel method for introducing DNA and modificating the native plasmid to allow effective selection of transformants has enabled the development of key molecular tools for studying Chlamydia. This transformation system was employed to test the expression of a diverse set of fluorescent proteins and to demonstrate the utility of subcellular localization studies [52]. The C. trachomatis developmental cycle incorporates numerous poorly understood processes, but a method for transforming Chlamydia has recently enabled the development of essential molecular tools to better study the biology and pathogenesis of these bacteria. Evaluation of green fluorescent protein (GFP) expression demonstrated a tight control of gene regulation by the Tetracycline repressor and anhydrotetracycline. Sensible GFP induction was observed in mid stages of the developmental cycle, data suggesting that metabolic diversity is affecting induction and/or expression during later stages [53].

Previously expensive, laborious and largely inaccessible, routine identification of tetracycline resistance loci became possible through the high-throughput whole-genome sequencing technology that emerged in recent years and enabled the low-cost interrogation of a large number of C. trachomatis genomes [54]. 
N. gonorrhoeae has quite a history in acquiring or developing resistance to antibiotics, including all first-line treatment drugs such as sulphonamides, penicillins, tetracyclines, macrolides, fluoroquinolones or, more recently, cephalosporins. Mechanisms for N. gonorrhoeae resistance to antibiotics may involve efflux systems, mutations in the chromosomal targets or acquisition of novel genes by transformation or plasmid-mediated conjugation. Chromosomal mosaic pen $A$ genes have been recently identified to confer resistance to extended-spectrum cephalosporins including ceftriaxone and cefixime, posing serious threats regarding the development of an untreatable 'superbug' [55].

In bacteria with multiple rRNA ( $\mathrm{rr}$ ) operons, the effect of a beneficial mutation in one operon is likely to be diluted out, thus offering no significant phenotypic advantage. However, amplification of a mutant allele could confer a resistant phenotype, as it occupies the majority of the bacterium's rrn operons. While implementation of both of these latter systems requires the acquisition of exogenous genetic material, moderate levels of macrolide resistance have been observed in N. gonorrhoeae upon overexpression of an endogenous membrane transport system. The potential risks of resistance developing via modification of endogenous efflux systems such as $m$ trRCDE of $N$. gonorrhoeae or via drug inactivation remain to be assessed [56].

A wide range of plasmids, from the small $4.2 \mathrm{~kb}$ plasmid to larger conjugative plasmids promoting their own transfer between strains and carrying determinants of antibiotic resistance, is associated with $N$. gonorrhoeae strains [6].

The use of molecular assays for gonococcal antimicrobial resistance surveillance is particularly suited to the fluoroquinolones, where mutations occur at defined bases in the quinolone resistance-determining regions (QRDRs) of the N. gonorrhoeae gyrA and parC genes. A realtime PCR (RT-PCR) assay was modified to allow the simultaneous detection of N.gonorrhoeae and quantification of the gonococcal susceptibility to ciprofloxacin using clinical samples, the assay being validated with the use of DNA extracted from 40 linked isolates and urethral swabs. The 40 isolates consisted of 11 susceptible isolates, 2 isolates of intermediate susceptibility and 27 resistant isolates. Twenty-four of these linked first-pass urine samples were obtained from men presenting with urethral gonorrhoea [8]. Each gonococcal isolate in the study was reidentified based on Gram staining - a positive oxidase test (Davies Diagnostics, Randburg, South Africa) and coagglutination with the Phadebact ${ }^{\circledR}$ Monoclonal Antibody Test (Pharmacia Diagnostics AB, Uppsala, Sweden). Ciprofloxacin-susceptibility profiles were confirmed using Etest-based MIC assays, while ciprofloxacin susceptibility phenotypes were determined according to Clinical and Laboratory Standards Institute (CLSI) guidelines.

DNA was extracted from an additional 33 first-pass urine specimens collected from patients. A previously published RT-PCR assay differentiating ciprofloxacin susceptible from nonsusceptible $N$. gonorrhoeae was modified by inclusion of primers and a probe to detect the gonococcal cytosine-specific DNA methyltransferase gene $(\mathrm{dcmH})$ as a positive control target. To generate gyrA and parC QRDR products for sequencing, amplification of the required sequences was undertaken using isolate-derived DNA. The RT-PCR assay correctly predicted that 15/33 samples were associated with ciprofloxacin-susceptible gonorrhoea, as both gyr $A$ and $\operatorname{par} C$ amplicons were produced. The 18 samples in which gyr $A$ amplicons were not generated were correctly predicted to come from patients with gonorrhoea either of intermediate 
susceptibility or resistant to ciprofloxacin. The $18 / 33$ samples that produced parC amplicons came from 15 patients with ciprofloxacin-susceptible gonorrhoea, 2 patients infected by N. gonorrhoeae isolates with intermediate susceptibility to ciprofloxacin and 1 patient with ciprofloxacin-resistant gonorrhoea. DNA extracted from first-pass urine of 15 out of 16 patients with ciprofloxacin-resistant gonorrhoea generated no gyr $A$ and $\operatorname{par} C$ amplicons. The modified RT-PCR assay previously described in this paper offered a faster method for combined N. gonorrhoeae diagnosis and determination of ciprofloxacin susceptibility than traditional culture and antimicrobial resistance testing [8].

As infection with Neisseria gonorrhoeae is one of the major causes of STDs in Romania, we conducted a study on bacterial gyrA and $\operatorname{par} C$ genes to determine the presence of ciprofloxacinresistant N. gonorrhoeae strains. About 200 subjects from Cluj-Napoca (Romania) suspected of STD infections were enrolled in our study. DNA extracted from first void urine samples was purified and amplified via multiplex PCR for the simultaneous detection of 6 STDs: Trichomonas vaginalis, M. hominis, M. genitalium, C. trachomatis, N. gonorrhoeae and U. urealyticum. DNA obtained from N. gonorrhoeae positive samples was amplified using gyrA, parC and $N$. gonorrhoeae-specific $d c m H$ gene primers designed using a modified version of previously published sequences [8]. DNA from a N. gonorrhoeae ATCC 49226 strain (sensible to ciprofloxacin) served as positive control. The amplicons were separated via gel electrophoresis (Figure 1).

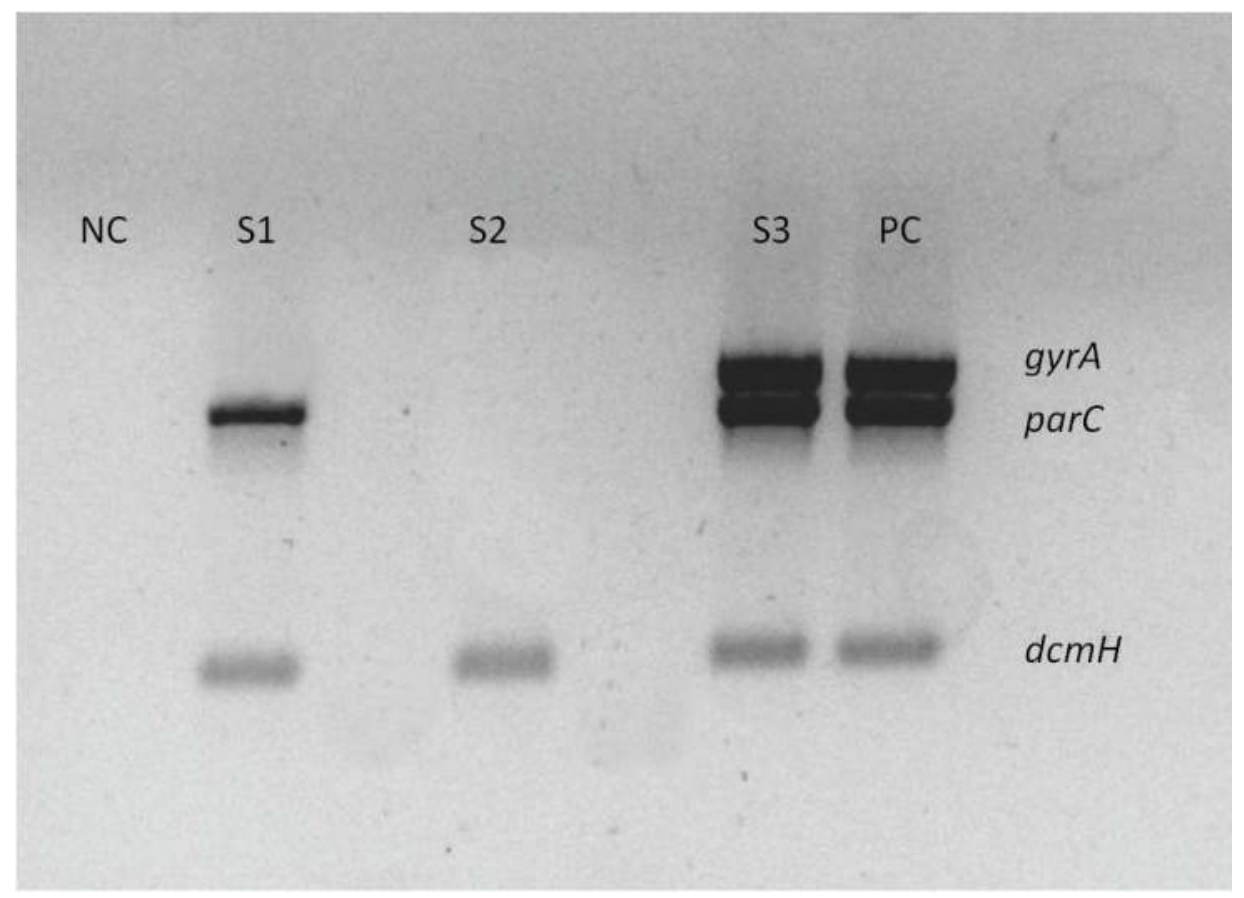

Figure 1. GyrA, parC and $d c m H$ genes-agarose gel electrophoresis. NC: negative control, S1-S3: samples, PC: positive control. 
The $d c m H$ gene was amplified in all of the samples collected from the 15 (7.5\%) patients diagnosed with gonorrhea, confirming the accuracy of both PCR methods employed in the study. The gyr $A$ and parC genes were amplified in eight samples accounting for ciprofloxacinsensitive strains (e.g., Sample S3 in Figure 1). The two genes were also present in the positive control sensitive to ciprofloxacin, proving that the primers were correctly designed. The gyr $A$ gene was not amplified in six samples presenting only the $\operatorname{par} C$ and $d c m H$ genes, that is, constituting intermediate-resistant strains (e.g., Sample S1 in Figure 1). One sample (Sample S2 in Figure 1) generated neither gyr A nor parC amplicons, signifying that the strain was resistant to ciprofloxacin.

This was the first Romanian study on STD pathogens demonstrating the presence of genes providing antibiotic resistance in the $N$. gonorrhoeae strains. The study confirmed the presence of $N$. gonorrhoeae strains resistant to ciprofloxacin in Romania. Most of the strains detected were intermediate-resistant ones.

Elsewhere, mutations at various genes such as $23 \mathrm{~S}$ rRNA, $m$ trR and penA (encoding penicillin-binding protein 2, PB P2) have been identified to associate with chromosomally mediated resistance to azithromycin (AZM) [57] and extended-spectrum cephalosporins [58] in N. gonorrhoeae.

A Chinese study combined antimicrobial susceptibility determinations with molecularbased analysis of AZM-resistant in N. gonorrhoeae according to the WHO recommendations. Bacterial genome DNA was extracted from each AZM-resistant isolate identified, mutations in the four alleles of the $23 \mathrm{~S}$ rRNA, $m \operatorname{tr} R$ and pen $A$ genes, as well as polymorphisms in por $B$ and $t b p B$, being amplified $[59,60]$.

A high potential for macrolide resistance to occur by mutations in the $23 \mathrm{~S}$ rRNAs of the bacteria was predicted. The target site for macrolides is the large (50S) subunit of the bacterial ribosome. Many cases of macrolide resistance in clinical strains can be linked to the alteration of specific nucleotides in $23 \mathrm{~S}$ rRNA within the large ribosomal subunit [10]. Findings were that single base substitutions in rRNA provide macrolide resistance [61].

About 2000 women were tested for vaginal infections. About 1536 of the 2000 vaginal swab samples cultivated in the study [4] were infected with at least one microorganism, a positivity rate of $76.80 \%$. M. hominis and U. urealyticum combined accounted for $<7 \%$. The antimicrobial tests were conducted on Muller agar medium, and antimicrobial activities were evaluated by measuring the diameters of inhibition around the disks. M. hominis strains were found to be more resistant than those of $U$. urealyticum.

In a 65-month study conducted on 373 patients attending the Clinic of Dermatovenereology in Belgrade from January 2007 to May 2012, urethral and cervical swabs were analyzed for the presence of $U$. urealyticum and M. hominis. About 48 (12.9\%) of the 373 specimens tested were positive for urogenital mycoplasmas: $37(77.8 \%)$ were found to be positive for U. urealyticum and $6(22.9 \%)$ for $M$. hominis, while $5(10.4 \%)$ were positive for both. The test also provided information regarding the density of each organism and its susceptibility to doxycycline, josamycin, ofloxacin, erythromycin, tetracycline, ciprofloxacin, azithromycin, clarithromycin and pristinamycin $[62,63]$. 
Among the 37 isolates of $U$. urealyticum, 34 (94.6\%) were sensitive to doxycycline. Furthermore, josamycin and azithromycin (70.3 and $67.6 \%$, respectively) were found to be highly and moderately potent against $U$. urealyticum. M. hominis was sensitive to doxycycline $(83.3 \%)$ and moderately sensitive to ofloxacin (66.7\%). M. hominis exhibited an absolute (100\%) drug resistance rate to erythromycin, tetracycline and clarithromycin, while $U$. urealyticum was highly resistant to clarithromycin $(94.6 \%)$, tetracycline $(86.5 \%)$, ciprofloxacin $(83.8 \%)$ and erythromycin (83.8\%). Despite being the most commonly used antibiotic in the treatment of nongonococcal genitourinary infections, doxycycline continues to be the most effective agent for $U$. urealyticum and M. hominis. Doxycycline and josamycin were the most potent antibiotics $(80 \%)$ in mixed infections with both U. urealyticum and M. hominis. Based on its effectiveness, josamycin could prove to be an alternative treatment for $U$. urealyticum infections. Still, it is believed that it is a matter of time until both mycoplasmas will develop resistance to these new generation antibiotics because of cross-border interaction with resistant agents [62].

Despite the evidence, physicians routinely favor macrolide antibiotics, for example, erythromycin when treating Ureaplasma infections in premature neonates [64].

Antibiotic resistance determination of Ureaplasma spp. usually requires predetermination of bacterial titer, followed by antibiotic interrogation using a set bacterial input. A method for determining precise MICs and one for screening against multiple antibiotics using breakpoint thresholds were detailed in another study [65] enabling a concurrent determination of the bacterial load in a sample simultaneously with the determination of resistance without prior knowledge of bacterial load. Further PCR and sequence analyses were performed to determine the resistance mechanism upon ureaplasmas grown in commercially available selective medium. An adapted breakpoint analysis was employed to screen for the presence of resistant mutants within the 61 isolates. Confirmation of Ureaplasma was determined by amplification of the Ureaplasma-specific urease gene (a 430-bp DNA product).

A reliable method to determine antimicrobial susceptibility of Ureaplasma isolates without prior knowledge of inoculum size was developed when mutations in bacterial genes previously associated with resistance were investigated for isolates resistant to erythromycin and ciprofloxacin [66]. DNA encoding bacterial 23S rRNA gene, L22 and L4 proteins were amplified and sequenced for macrolide-resistant strains. Gyrase subunits and topoisomerase subunits were sequenced for ciprofloxacin-resistant strains, while tet $M$ genes from both tetracycline-susceptible and tetracycline-resistant isolates were also sequenced. More erythromycin-resistant studies are required to determine the prevalence of mutations in the relevant genes associated with resistance.

\section{Conclusions}

Discovery of antibiotic resistant strains is an important step in choosing the appropriate treatment against bacterial STD after the positive detection of the causative agents. A proper treatment can prevent the spreading of these diseases in the general population. Antimicrobial susceptibility testing proves to be useful in cases when bacteria can be isolated. 
The antibiotic resistance of $C$. trachomatis has significantly different characteristics from those of other bacteria. First, because chlamydiae are intracellular pathogens, antimicrobial susceptibility must be determined by their ability to proliferate within a host cell in the presence of variable antibiotic concentrations. Second, in contrast to most bacteria, C. trachomatis displays a "heterotypic resistance" in vitro, the chlamydial population containing both susceptible and resistant organisms, only a small proportion of them exerting resistance at any given time [17].

The study of the Chlamydiae biology is complicated by their intracellular development, but knowledge of the recombination mechanism(s) may enable effective genetic manipulations.

Antimicrobial resistance of microorganisms is a quite dynamic phenomenon, highlighting the need for current prevalence and susceptibility data from various different institutions or geographic areas. The ability to use molecular assays for STD antimicrobial resistance surveillance would offer a significant advantage to public health programs and selected patient management.

Drug therapies can result in strains containing mutations that confer the highest resistance becoming prevalent. In contrast, rRNA mutations in laboratory strains are intentionally created in order to evaluate drug interaction mechanisms. The creation of phenotypes with less effective resistance is possible only under controlled laboratory conditions.

The routine use of genomics in clinical settings is becoming a reality. For STDs, understanding the diversity of the circulating strains and their changing over time pattern is a primary requirement. Comparative genomic studies on chlamydia have demonstrated that it undergoes extensive recombination. Genome projects can also help us understand the phenomenon of antibiotic resistance in STD agents, particularly in the cases of C. trachomatis, where very little was known about the genetics of these organisms because of a lack of molecular tools and the inability to grow them outside cell cultures or host organisms [14].

In recent year, genomics was asked to clarify a number of clinical aspects. Although genome analysis of bacterial STD was not a main issue, it can undoubtedly reveal previously inaccessible aspects of pathogen biology, including changes that lead to antibiotic resistance thereof. Genetic methods enable the DNA extraction from different biological samples, and both the presence of the bacteria and its resistance to one or more antibiotics can be determined from a single DNA sample. By studying the genes that induce antibiotic resistance and the plasmids that transfer such genes the mechanism that leads to antibiotic resistance can be elucidated.

\section{Author details}

Vică Mihaela Laura ${ }^{1 *}$, Matei Horea Vladi ${ }^{1}$ and Siserman Costel Vasile ${ }^{2}$

*Address all correspondence to: mvica@umfcluj.ro

1 Department of Cell and Molecular Biology, "Iuliu Haţieganu” University of Medicine and Pharmacy, Cluj-Napoca, Romania

2 Department of Legal Medicine, "Iuliu Hațieganu" University of Medicine and Pharmacy, Cluj-Napoca, Romania 


\section{References}

[1] Nwadioha S., Egesie J.O., Emejuo H., Iheanacho E. A study of female genital swabs in a Nigerian Tertiary Hospital. Asian Pac J Trop Med. 2010;3:577-579.

[2] Ekabua J.E., Agan T.U., Iklaki C.U., Ekanew E.I. Adjuncts to case assessment of vaginal discharge syndrome in pregnant women. Asian Pac J Trop Med. 2010;3:63-65.

[3] Simpore J., Ilboudo D., Karou D., Pietra V., Granato M., Esposito M., et al. Prevalence of HHV-8 infections associated with HIV, HBV and HCV in pregnant women in Burkina Faso. J Med Sci. 2006;6:93-98.

[4] Karou SD, Djigma F, Sagna T., Nadembega C., Zeba M., Kabre A., Anani K., Ouermi D., et al. Antimicrobial resistance of abnormal vaginal discharges microorganisms in Ouagadougou, Burkina Faso. Asian Pac J Trop Biomed. 2012;2(4):294-297.

[5] Pawlikowska-Warych M., Śliwa-Dominiak J., Deptuła W. Chlamydial plasmids and bacteriophages. Acta Biochimica Polonia. 2015;62(1):1-6. doi:10.18388/abp.2014_764

[6] Snyder L.A.S., Davies J.K., Ryan C.S., Saunders N.J. Comparative overview of the genomic and genetic differences between the pathogenic Neisseria strains and species. Plasmid. 2005;54:191-218.

[7] Samra Z., Rosenberg S., Soffer Y., Dan M. In vitro susceptibility of recent clinical isolates of Chlamydia trachomatis to macrolides and tetracyclines. Diagn Microbiol Infect Dis. 2001;39:177-179.

[8] Magooa M.P., Müller E.E., Gumede L., Lewis D.A. Determination of Neisseria gonorrhoeae susceptibility to ciprofloxacin in clinical specimens from men using a real-time PCR assay. Int J Antimicrob Ag. 2013;42:63-67.

[9] Unemo M., Shafer W.M. Antimicrobial resistance in Neisseria gonorrhoeae in the 21st century: past, evolution, and future. Clin Microbiol Rev. 2014;27:587-613.

[10] Vester B., Douthwaite S. Macrolide resistance conferred by base substitutions in 235 rRNA. Antimicrob Agents Chemother. 2001;45:1-12.

[11] Schlunzen F., Zarivach R., Harms J., Bashan A., Tocilj A., Albrecht R., et al. Structural basis for the interaction of antibiotics with the peptidyl transferase centre in eubacteria. Nature. 2001;413:814-821.

[12] Misyurina O.Y., Chipitsyna E.V., Finashutina Y.P., Lazarev V.N., Akopian T.A., Savicheva A.M., et al. Mutations in a $23 \mathrm{~S}$ rRNA gene of Chlamydia trachomatis associated with resistance to macrolides. Antimicrob Agents Chemother. 2004;48(4):1347-1349.

[13] Jiang Y., Zhu H., Yang L.N., Liu Y.J., Hou S.P., Qi M.L., et al. Differences in 23 S ribosomal RNA mutations between wild-type and mutant macrolide-resistant Chlamydia trachomatis isolates. Exp Ther Med. 2015;10:1189-1193. doi:10.3892/etm.2015.2595 
[14] Seth-Smith H.B.M., Thomson N.R. Whole-genome sequencing of bacterial sexually transmitted infections: implications for clinicians. Curr Opin Infect Dis. 2013;26:90-98. doi:10.1097/QCO.0b013e32835c2159

[15] Dean D. Pathogenesis of chlamydial ocular infections. In: K. Tasman and E. Jaeger, editors. Duane's Foundations of Clinical Ophthalmology, vol 2. Philadelphia, PA: Lippincott Williams \& Wilkins; 2002. pp. 1-20.

[16] Dugan J., Rockey D.D., Jones L., Andersen A.A. Tetracycline resistance in Chlamydia suis mediated by genomic islands inserted into the chlamydial inv-like gene. Antimicrob Agents Chemother. 2004;48:3989-3995. doi:10.1128/AAC.48.10.3989-3995.2004

[17] Somani J., Bhullar V.B., Workowski K.A., Farshy C.E., Black C.M. Multiple drug-resistant Chlamydia trachomatis associated with clinical treatment failure. J Infect Dis. 2000;181:1421-1427.

[18] Suchland R.J., Geisler W.M., Stamm W.E. Methodologies and cell lines used for antimicrobial susceptibility testing of Chlamydia spp. Antimicrob Agents Chemother. 2003;47:636-642.

[19] Blythe M.J., Katz B.P., Batteiger B.E., Ganser J.A., Jones R.B. Recurrent genitourinary chlamydial infections in sexually active female adolescents. J Pediatr. 1992;121:487-493.

[20] Munday P.E., Thomas B.J., Gilroy C.B., Gilchrist C., Taylor-Robinson D. Infrequent detection of Chlamydia trachomatis in a longitudinal study of women with treated cervical infection. Genitourin Med. 1995;71:24-26.

[21] Grayston J.T., Wang S.P. New knowledge of chlamydiae and the diseases they cause. J Infect Dis. 1975;132:87-105.

[22] Hogan R.J., Mathews S.A., Mukhopadhyay S., Summersgill J.T., Timms P. Chlamydial persistence: beyond the biphasic paradigm. Infect Immun. 2004;72:1843-1855.

[23] Mpiga P., Ravaoarinoro M. Chlamydia trachomatis persistence: an update. Microbiol Res. 2006;161:9-19.

[24] Senn L., Hammerschlag M.R., Greub G. Therapeutic approaches to Chlamydia infections. Expert Opin Pharmacother. 2005;6:2281-2290.

[25] Horner P. The case for further treatment studies of uncomplicated genital Chlamydia trachomatis infection. Sex Transm Infect. 2006;82:340-343.

[26] Shepard M.C. The recovery of pleuropneumonia-like organisms from Negro men with and without nongonococcal urethritis. Am J Syph Gonorrhea Vener Dis. 1954;38:113-124.

[27] Pararas M.V., Skevaki C.L., Kafetzis D.A. Preterm birth due to maternal infection: causative pathogens and modes of prevention. Eur J Clin Microbiol Infect Dis. 2006;25:562-569.

[28] Kenny GE., Cartwright F.D. Susceptibilities of Mycoplasma hominis, M. pneumoniae, and Ureaplasma urealyticum to GAR-936, dalfopristin, dirithromycin, evernimicin, gatifloxacin, linezolid, moxifloxacin, quinupristin-dalfopristin, and telithromycin compared to 
their susceptibilities to reference macrolides, tetracyclines, and quinolones. Antimicrob Agents Chemother. 2001;45:2604-2608.

[29] Baseman J.B., Dallo S.F., Tully J.G., Rose D.L. Isolation and characterization of Mycoplasma genitalium strains from the human respiratory tract. J Clin Microbiol. 1988;26:2266-2269.

[30] BjorneliusE.,LidbrinkP.,JensenJ.S.Mycoplasmagenitaliuminnon-gonococcalurethritisa study in Swedish male STD patients. Int J STD AIDS. 2000;11:292-296.

[31] Deguchi T., Maeda S. Mycoplasma genitalium: another important pathogen of nongonococcal urethritis. J Urol. 2002;167:1210-1217.

[32] Tully J., Taylor-Robinson D., Rose D.L., Cole R.M., Bove J.M. Mycoplasma genitalium, a new species from the human urogenital tract. Int J Syst Bacteriol. 1983;33:387-396.

[33] Koutsky L.A., Stamm W.E., Brunham R.C., Stevens C.E., Cole B., Hale J., et al. Persistence of Mycoplasma hominis after therapy: Importance of tetracycline resistance and of coexisting vaginal flora. Sex Transm Dis. 1983;10:374-381.

[34] Mardassi B.B., Aissani N., Moalla I., Dhahri D., Dridi A., Mlik B. Evidence for the predominance of a single tet(M) gene sequence type in tetracycline-resistant Ureaplasma paroum and Mycoplasma hominis isolates from Tunisian patients. J Med Microbiol. 2012;61:1254-1261.

[35] Farkas B., Ostorházi E., Pónyai K., Tóth B., Adlan E., Párducz L., et al. Frequency and antibiotic resistance of Ureaplasma urealyticum and Mycoplasma hominis in genital samples of sexually active individuals. Orv Hetil. 2011;152:1698-1702.

[36] Karabay O., Topcuoglu A., Kocoglu E., Gurel S., Gurel H., Ince N.K. Prevalence and antibiotic susceptibility of genital Mycoplasma hominis and Ureaplasma urealyticum in a university hospital in Turkey. Clin Exp Obstet Gynecol. 2006;33:36-38.

[37] Waites K.B., Crouse D.T., Cassell G.H. Therapeutic considerations for Ureaplasma urealyticum infections in neonates. Clin Infect Dis. 1993;17(Suppl. 1):208-214.

[38] Suchland R.J., Sandoz K.M., Jeffrey B.M., Stamm W.E., Rockey D.D. Horizontal transfer of tetracycline resistance among Chlamydia spp. in vitro. Antimicrob Agents Chemother. 2009;53:4604-4611.

[39] Norris S.J., Cox D.L., Weinstock G.M. Biology of Treponema pallidum: correlation of functional activities with genome sequence data. J Mol Microbiol Biotechnol. 2001;3:37-62.

[40] Leclercq R., Courvalin P. Bacterial resistance to macrolide, lincosamide, and streptogramin antibiotics by target modification. Antimicrob Agents Chemother. 1991;35:1267-1272.

[41] Robert DeMars R., Weinfurter J. Interstrain gene transfer in Chlamydia trachomatis in vitro: mechanism and significance. J Bacteriol. 2008;190(5):1605-1614.

[42] Srinivasan T., Bruno W.J., Wan R., Yen A., Duong J., Deborah Dean D. In Vitro Recombinants of antibiotic-resistant Chlamydia trachomatis strains have statistically more breakpoints 
than clinical recombinants for the same sequenced loci and exhibit selection at unexpected loci. J Bacteriol. 2012;194(3):617-626. doi:10.1128/JB.06268-11

[43] Millman K., Black C.M., Johnson R.E., Stamm W.E., Jones R.B., Hook E.W., et al. Population based genetic and evolutionary analysis of Chlamydia trachomatis urogenital strain variation in the United States. J Bacteriol. 2004;186:2457-2465.

[44] Ortiz L., Angevine M., Kim S.K., Watkins D., DeMars R. T-cell epitopes in variable segments of Chlamydia trachomatis major outer membrane protein elicit serovar-specific immune responses in infected humans. Infect Immun. 2000;68:1719-1723.

[45] Dean D., Millman K. Molecular and mutation trends analyses of omp1 alleles for serovar E of Chlamydia trachomatis. Implications for the immunopathogenesis of disease. J Clin Investig. 1997;99:475-483.

[46] Sayada C., Denamur E, Grandchamp B., Orfila J., Elion J. Denaturing gradient gel electrophoresis analysis for the detection of point mutations in the Chlamydia trachomatis major outer-membrane protein gene. J Med Microbiol. 1995;43:14-25.

[47] Binet R., Maurelli A.T. Frequency of development and associated physiological cost of azithromycin resistance in Chlamydia psittaci 6BC and C. trachomatis L2. Antimicrob Agents Chemother. 2007;51:4267-4275.

[48] Rice R.J., Bhullar V., Mitchell S.H., Bullard J., Knapp J.S. Susceptibilities of Chlamydia trachomatis isolates causing ncomplicated female genital tract infections and pelvic inflammatory disease. Antimicrob Agents Chemother. 1995;39:760-762.

[49] Kahan F.M., Kahan J.S., Cassidy P.J., Kropp H. The mechanism of action of fosfomycin (phosphonomycin). Ann N Y Acad Sci. 1974;235:364-386.

[50] McCoy A.J., Sandlin R.C., Maurelli A.T. In vitro and in vivo functional activity of Chlamydia MurA, a UDP-N-Acetylglucosamine enolpyruvyl transferase involved in peptidoglycan synthesis and fosfomycin resistance. J Bacteriol. 2003;185(4):1218-1228.

[51] O'Neill C.E., Seth-Smith H.M.B., Van Der Pol B., Harris S.R., Thomson N.R., Cutcliffe L.T., et al. Chlamydia trachomatis clinical isolates identified as tetracycline resistant do not exhibit resistance in vitro: whole-genome sequencing reveals a mutation in porB but no evidence for tetracycline resistance genes. Microbiology. 2013;159:748-756.

[52] Agaisse H., Derré I. A C. trachomatis cloning vector and the generation of C. trachomatis strains expressing fluorescent proteins under the control of a C. trachomatis promoter. PLoS One. 2013;8:e57090. doi:10.1371/journal.pone.0057090

[53] Wickstrum J., Sammons L.R., Restivo K.N., Hefty P.S. Gene expression in Chlamydia trachomatis using the Tet system. PLoS One. 2013;8(10):e76743.

[54] Harris S.R., Clarke I.N., Seth-Smith H.M., Solomon A.W., Cutcliffe L.T., Marsh P., et al. Whole-genome analysis of diverse Chlamydia trachomatis strains identifies phylogenetic relationships masked by current clinical typing. Nat Genet. 2012;44(S1):413-419. 
[55] Unemo M., Shafer W.M. Antibiotic resistance in Neisseria gonorrhoeae: origin, evolution and lessons learned for the future. Ann N Y Acad Sci. 2011;1230:19-28.

[56] Hagman K.E., Pan W., Spratt B.G., Balthazar J.T., Judd R.C., Shafer W.M. Resistance of Neisseria gonorrhoeae to antimicrobial hydrophobic agents is modulated by the mtrRCDE efflux system. Microbiology. 1995;141:611-622.

[57] Chisholm S.A., Dave J., Ison C.A. High-level azithromycin resistance occurs in Neisseria gonorrhoeae as a result of a single point mutation in the $23 \mathrm{~S}$ rRNA genes. Antimicrob Agents Chemother. 2010;54(9):3812-3816.

[58] Liao M., Gu W.M., Yang Y., Dillon J.A. Analysis of mutations in multiple loci of Neisseria gonorrhoeae isolates reveals effects of PIB, PBP2 and MtrR on reduced susceptibility to ceftriaxone. J Antimicrob Chemother. 2011;66(5):1016-1023.

[59] Liang J.Y., Cao W.L., Li X.D., Bi C., Yang R.D., Liang Y.H., et al. Azithromycin-resistant Neisseria gonorrhoeae isolates in Guangzhou, China (2009-2013): coevolution with decreased susceptibilities to ceftriaxone and genetic characteristics. BMC Infect Dis. 2016;16:152-159. doi:10.1186/s12879-016-1469-3

[60] World Health Organization. Global action plan to control the spread and impact of antimicrobial resistance in Neisseria gonorrhoeae. 2012.[Internet]. 2012. Available from: http:// www.who.int/reproductivehealth/publications/rtis/9789241503501/en/ [Accessed: 21 October 2016].

[61] Sor F., Fukuhara H. Identification of two erythromycin resistance mutations in the mitochondrial gene coding for the large ribosomal RNA in yeast. Nucleic Acids Res. 1982;10:6571-6577.

[62] Skiljevic D., Mirkov D., Vukicevic J. Prevalence and antibiotic susceptibility of Mycoplasma hominis and Ureaplasma urealyticum in genital samples collected over 6 years at a Serbian university hospital. Indian J Dermatol Venereol Leprol. 2016;82:37-41.

[63] Clegg A., Passey M., Yoannes M., Michael A. High rates of genital Mycoplasma infection in the highlands of Papua New Guinea determined both by culture and by a commercial detection kit. J Clin Microbiol. 1997;35:197-200.

[64] Matlow A., Th'ng C., Kovach D., Quinn P., Dunn M., Wang E. Susceptibilities of neonatal respiratory isolates of Ureaplasma urealyticum to antimicrobial agents. Antimicrob Agents Chemother. 1998;42:1290-1292.

[65] Beeton M.L., Chalker V.J., Maxwell N.C., Kotecha S., Spiller O.B. Concurrent titration and determination of antibiotic resistance in Ureaplasma species with identification of novel point mutations in genes associated with resistance. Antimicrob Agents Chemother. 2009;53(5):2020-2027.

[66] Bebear C.M., Renaudin H., Charron A., Gruson D., Lefrancois M., Bebear C. In vitro activity of trovafloxacin compared to those of five antimicrobials against mycoplasmas including Mycoplasma hominis and Ureaplasma urealyticum fluoroquinolone-resistant isolates that have been genetically characterized. Antimicrob Agents Chemother. 2000;44:2557-2560. 
